\title{
Comparación in vitro de la estabilidad y exactitud dimensional entre tres marcas de siliconas por condensación ${ }^{1}$
}

\author{
In Vitwo Comparison Of Stability And Dimensional \\ Exactitude Among Three Brands Of Silicone By \\ Condensation
}

Abstract

$E$ In la presente imvestigacioin se comparó la estabitidad y exactizud dimenronal de tres materiales de impresión lipo siticona por condensación Speedex, Colloflax y Protesil en tres medidas (dos diámelros y una longitud) con respecto a un modelo metálico. Se tomaron del modelo melálico 30 impresiones, 10 por cada marca comercial de silicona tuego se vaciann con yeso exiraduro y se tomaron Las medidas con un micrómetro en 3 dimensiones (MI), $C O, V L$ ). De los resultados oblenidos se concluye que las tres siticonas presentan diferente exactitud dimensional.

Palabras dave: Fstabilidad dimensional, Exactitud dimensional, Siticonas por condensación.
Artero Arévalo, Rafael rafart12@hotmail.com Bonilla Alvarado, fosé calzon90@hotmail.com Campos Rodriguez, Teddy teddytotal.90yahoo.com

Egresados de la Carrera Doctorado en Cirugia Dental 


\section{Introducción}

Los materiales dentales han sido investigados desde el siglo XVI como entidad odontológica; Poller A. mencionado por Anusavice, (2001) descubrió los primeros materiales de impresión tipo hidrocoloides reversibles "agar".

La función de un material de impresión consiste en registrar con exactitud las dimensiones de los tejidos bucales y sus relaciones especiales. Para obtener una impresión, se aplica en los tejidos orales un material en estado plástico para que frague. (Orellana Velasco, 1987)

Debido a la alteración en cuanto a la exactitud dimensional, encontradas en las impresiones definitivas para el área de prostodoncia fija, los adaptes de estructuras metálicas no son los deseados y esto significa repetir todo el procedimiento desde impresiones. La dificultad que se puede encontrar a corto, mediano y largo plazo si no se resuelve el problema de exactitud dimensional es desadapte de la prótesis, caries dental, sensibilidad, neerosis pulpar. problemas periodontales y oclusales.

La impresión dental es la reproducción en negativo de los tejidos duros y blandos de la cavidad bucal, de la cual se obticne una reproducción en positivo o modelo. (Tylman`s, 1991)

Para Tylman's la fabricación de un colado requiere de un material de impresión que reproduzca un negativo exacto de los tejidos orales y para esto es necesario que el material de impresión presente: Plasticidad total antes del fraguado, Fluidez suficiente para registrar detalle fino, Exactitud dimensional, Estabilidad dimensional y Elasticidad después del fraguado.

Las siliconas son materiales elásticos para impresiones a base de polidimetil o polivinil-siloxanos. Deben su nombre a la presencia de sílice y oxigeno en su composición. Se utilizan en impresiones para coronas y puentes fijos, en impresiones totales de paciente total o parcialmente edéntulos, para registro de mordida, en procedimientos de laboratorio para el procesado de prótesis totales y parciales. Estas pueden clasificarse de acuerdo a su consistencia y la composición de polimerización. De acuerdo a la consistencia se presentan en cuatro tipos: cuerpo liviano, regular, pesado, extrapesado o masilla. De acuerdo a la reacción de polimerización se clasifican en: Siliconas por adición y Siliconas por condensación Las siliconas por condensación son materiales elásticos a base de polidimetil siloxano, denominadas tambićn siliconas orgánicas de estaño por su catalizador, la reacción de condensación se produce por la eliminación del alcohol etílico. (Shillingburg, 2000).

En el mercado suelen encontrarse como una pasta de base y un liquido de baja viscosidad o pasta catalizadora. Debido a que el polimero es un líquido coloidal de sílice o de óxidos metálicos de tamaño pequeño, se agregan como relleno para formar una pasta. Respecto a sus propiedades la estabilidad dimensional y la reproducción de detalles son muy importantes. Al igual que los otros materiales para impresiones, las siliconas por condensación se contraen y esta contracción se debe a la polimerización del material, a la volatilización del alcohol que es un producto residual. La mayor contracción ocurre dentro de las primeras 24 horas. Respecto a la reproducción de detalles, estas siliconas reproducen detalles similares a la del agar-agar, mercaptanos y Poliéteres y mucho mejor que la de los alginatos. (Anusavice, 2001).

Con el material Speedex putty el momento ideal para el vaciado del modelo está entre los $30 \mathrm{~min}$. y las 72 horas tras haber sido tomada la impresión. Cion el Coltoflax el vaciado ideal va de los 30 minutos a las 72 horas tras la toma de impresión. Según el fabricante la estabilidad dimensional extraordinaria, es posible colar el modelo hasta 7 días tras la toma de impresión sin pérdida dimensional alguna. Con el Protesil Putty ; el momento ideal para el vaciado es de 30 minutos a 72 horas posterior a la polimerización del material

\section{Metodología de la Investigación}

Para llevar a cabo el estudio se confeccionó un modelo metálico (fig. 1 en forma de incisivo con diámetros de Mesio distal de $15.77 \mathrm{~mm}$, Vestíbulo lingual de $11.96 \mathrm{~mm}$ y cérvico oclusal de $18.64 \mathrm{~mm}$ en aleación de cromo cobalto, tomando como modelo un incisivo central superior de yeso piedra con todas las características anatómicas de un diente natural, llevando una base de $3 \mathrm{~cm}$. en el cual establecerá contacto con la cubeta individual, fabricada de $3 \mathrm{~cm}$. de ancho por $3 \mathrm{~cm}$. de largo y perforada para un mejor escurrimiento del material de impresión.

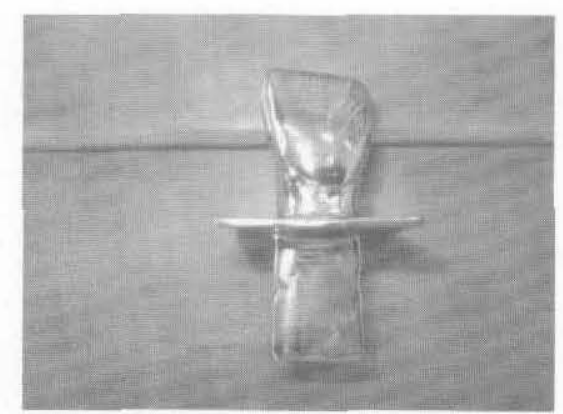

Figura 1. Modelo utilizado para impresiones.

Después se tomaron 10 impresiones con cada marca comercial de silicona por condensación "Speedex, Coltoflax de la casa Coltene/ Whaledent inc y Protesil de la casa Vannini Dental Industry", las cuales se mezclaron con la técnica que proporciona el fabricante por el tiempo indicado, se tomaron con una cubeta individual de metal (fig.2) por un solo operador y se vaciaron con yeso extraduro con las medidas exactas de agua y polvo (100gr. de yeso por $2.5 \mathrm{ml}$ de agua) con un tiempo de mezcla de 20 a 30 segundos dándole un tiempo de fraguado de 60 minutos a cada modelo. 


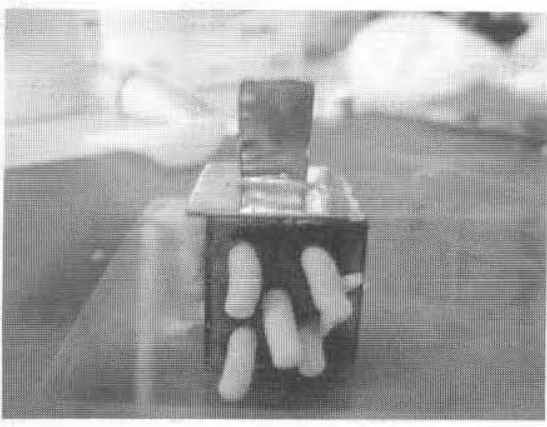

Figura 2. Cubeta individual metálica perforada

Se tomaron las mediciones con micrómetro (fig. 3) para obtener medidas mas exactas se rcalizaron en el área de ingeniería mecánica del ITCA las cuales se tomaron en 3 dimensiones VL, OC, MD para obtener mediciones mas exactas de cada uno de los modelos, al modelo metálico se le realizaron muescas a nivel de las 3 dimensiones, llenando una ficha proporcionada por el grupo al operador.

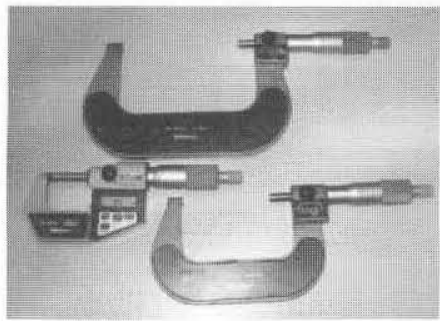

Figura3. Micrometro.

\section{Análisis de Resultados}

Los datos se organizaron de acuerdo a la marca comercial de silicona por condensación de los modelos de yeso extraduro y también por medio de la medición de las tres medidas obtenidas en los modelos "mesiodistal, vestíbulo-lingual y cervico-oclusal", se tabularon por medio del programa Lxcel de Office.

Comparación de las medias entre los modelos obtenidos y la medida del modelo control

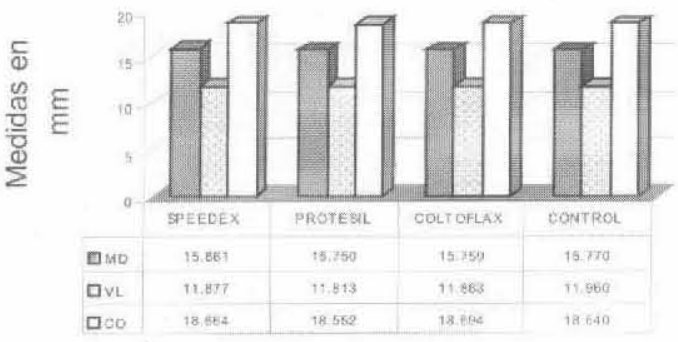

En el gráfico anterior se comparan las medias de los modelos de yeso obtenidos de las tres marcas comerciales con las medidas reales de control del modelo metálico, en las tres superficies M-D, V-L y
C-O, obscrvándose que las medidas del modelo obtenido a partir de la impresión con la silicona marca Speedex se acerca más a la medida del modelo metálico de control, por lo tanto, se puede decir que este material presenta mejor cstabilidad y exactitud dimensional que la marca Coltoflax y Protesil. Estadísticamente, estas diferencias entre las medias de cada material con el modelo metálico son estadísticamente significativas al 0.5. La prueba estadística aplicada fue la $\mathrm{t}$ para dos muestras suponiendo varianzas desiguales de las medias de cada material con respecto al modelo metálico de control con las medias obtenidas a partir de las medidas de C-O y V-L (Cervico Oclusal y Vestíbulo Lingual).

Esto difiere de lo encontrado en el Estudio comparativo in Vitro de la estabilidad dimensional entre una silicona por condensación speedex y otra por adición "elite" realizado en el año 2002 en Facultad de Odontología de la Universidad Corporativa de Colombia. En este trabajo se comparó la estabilidad dimensional de estos dos materiales en cuatro medidas (tres diámetros y una longitud) con respecto a un troquel maestro en diferentes tiempos de vaciado, mediante la toma de impresiones con cada material. El análisis estadístico de los resultados de este estudio demostró que ambas siliconas se asemejan considerablemente a las medidas del troquel maestro sugiriendo así que entre las medidas diámetro 1, diámetro 2 , diámetro 3 , no existen diferencias estadísticamente significativas, mientras que en la medida longitud 1 los resultados se aproximan a una posible diferencia entre el troquel maestro y los modelos obtenidos de los dos materiales de impresión.

\section{Conclusiones}

De acuerdo a los resultados obtenidos se establece que las marcas comerciales de silicona por condensacion Speedex, Protesil y Coltoflax, presentaron diferente estabilidad y exactitud dimensional en las tres superficies medidas en $\mathrm{mm}$ comparando con la medida control

Los modelos de yeso obtenidos con la marca de silicona por condensación Speedex presentaron mejor estabilidad y exactitud dimensional, comparando con la medida control del modelo metálico. Posterior a la investigación la marca comercial Speedex presentó mejor tiempo de trabajo; y una manipulación más favorable al operador tomando en cuenta todas las variantes de control

\section{Recomendaciones}

Utilizar la silicona por condensación marca Speedex, ya que de acuerdo a los resultados de este estudio es la que mejor mantiene la estabilidad y exactitud dimensional de las estructuras que son impresionadas con este material.

Al gremio odontológico y estudiantes de odontología, 
que las siliconas por condensación son materiales que presentan buena estabilidad y exactitud dimensional siempre y cuando se sigan las indicaciones del fabricante.

A la luz de los resultados antes mencionados se recomienda a los docentes instruir a los estudiantes sobre la necesidad de seguir las indicaciones de las diferentes marcas de silicona según el fabricante. Tener en cuenta todas las variantes como: la temperatura, manipulación del material, proporción polvo líquido; ya que a veces los malos resultados suelen estar asociados a estos y no a la calidad del material.

\section{Agradecimientos}

Dr. Carlos Roberto Ramos Menjüar por su asesoría en el contenido teórico, Dra. Elizabeth Pérez Otiva por asesoria en metodologia, Ing. Carlos Monrroy (ITCA) por toma de mediciones de los modelos de yeso y al Ing. Fabio Bautista Pérez por asesoría en estadística.

\section{Fuentes de Información Consultadas}

1.Anusaice, (2001) ciencia de los materiales dentales de, Phillip. $10^{a}$ Edicion, Editorial MG Graw-Hill interamericana. 2. Covan José Luis, $(2004)$ biomateriales dentales $I^{a}$ Edición 3. Facultad de Odontología de la Universidad Corporativa de Colombra.
Estudio Comparatio In Vitro de la Estabilidad dimensional enire una Silicona por Condensación "Speedex" y otra por Adición "Elite" en el Año 2002.

hitp://ucs.educo/columbus/cientificos/ciliconas/resultados.htm Consultado 9/4/2007.

4. Ficha técnica de silicona por condensación Speedex. 5. Ficha lécnica de silicona por condensación Protesil.

6. Ficha técnica de silicona por condensación Coltoflax.

7.Medeos(2005) Siticonas por condensación protesil En linea. Consultado 9 de abril del 2007. Disponible en hup://wres.bgdent.com

8. Odontocats (2000), Especialidades prótesis En linea consultado 9 abril del 2007. Disponible en http://wwis.odontocat.com/protesiscsal.htm

9. Schawartz, Richard, (1999) fundamentos en odontología operalorio $I^{a}$ Edición, Editorial LTDA.

10.Shillingburg Herbert T., (2000) fundamentos esenciales en prótesis fuja $3^{a}$ Edición, Editorial Qintessence S.L.

11. Tylman's William Malone, (1991) teoria y practica en prostodoncia fija $8^{a}$ Edición, Editorial actualidades medico odontológicas Latinoamérica.

12. Vargas, Omar A. (2002), Técnicas de materiales de impresión. En linea consultado 9 Abril del 2007. Disponible en http://wwew odontologos.com.co/SCODB/impresion. htm 13. Vigodent (2003) Speedex hidrofilito con estabilidad dimensional. En linea Consullado 9 abril del 2007 Disponible en hllp.zeww.vigodent.com. Br/speedex introducao.htm. 


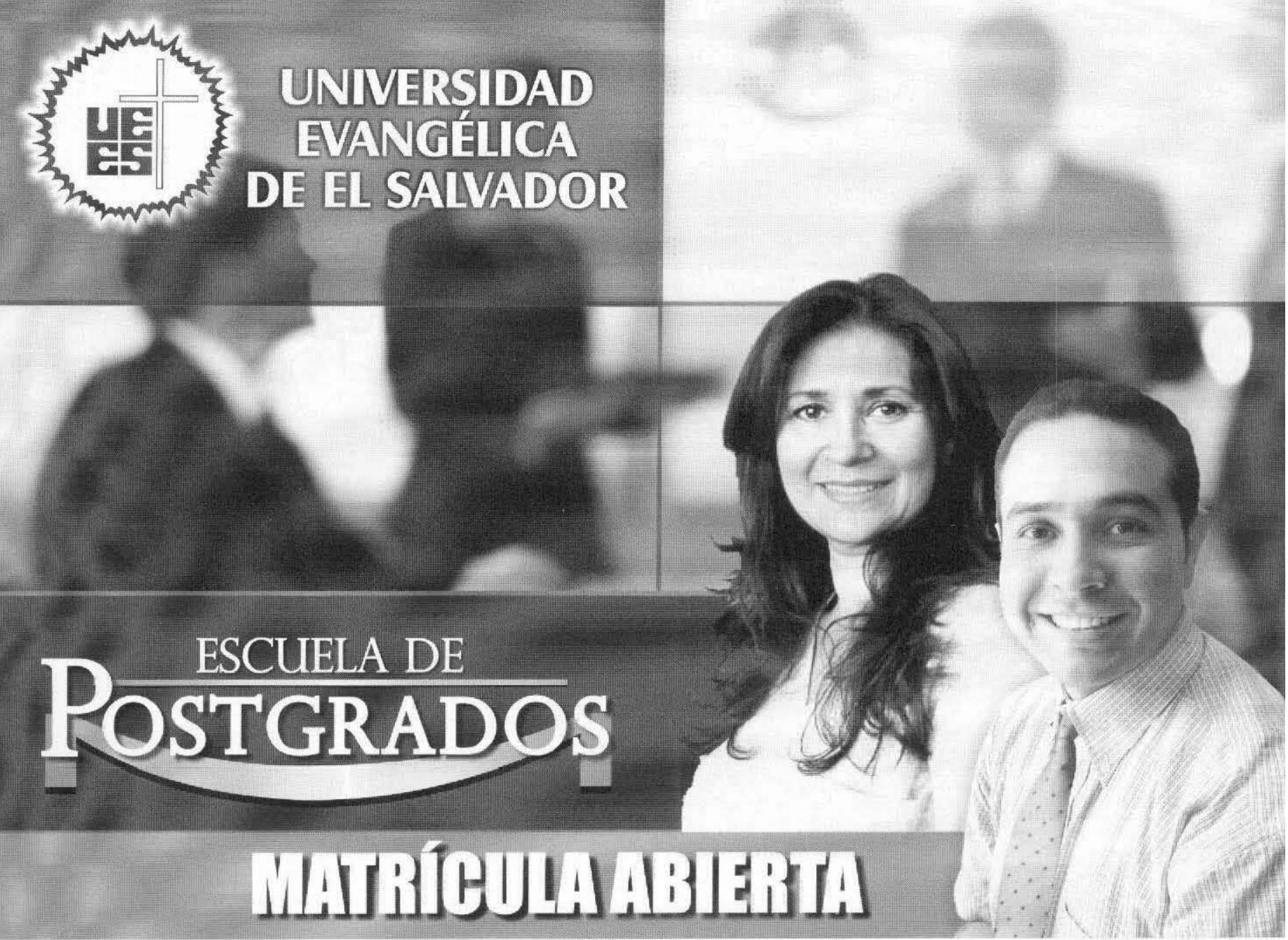

- MAESTRÍA EN DOCENCIA UNIVERSITARIA

DIRIGIDA A:

Todo profesional con vocación pedagógica comprometido con la realidad social y educativa y con una actitud positiva hacia el desempeño docente en el nivel educativo superior.

\section{- MAESTRÍA EN METODOLOGÍA DE LA INVESTIGACIÓN CIENTÍFICA}

\section{DIRIGIDAA:}

Los Profesionales en puestos clave de dirección, coordinación y gerencia, docentes e investigadores del nivel de educación secundariay superior y profesionales de los sectores de educación, salud, tecnología, economía, humanidades, ciencias sociales $y$ ciencias naturales.

\section{Tol: 2215-4032}

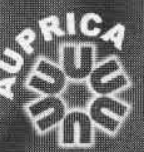

Aspectos académicos freddy. leistenschneider@uees.edu.sv

Aspectos administrativos sandra.grijalva@uees.edu.sv

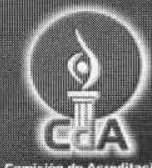

- MAESTRIÍA EN ADMINISTRACIÓN DE RECURSOS HUMANOS

\section{DIRIGIDA A:}

Profesionales interesados en ampliar sus competencias en la administración y gestión de los recursos humanos de organizaciones públicas, privadas, y oroanizaciones no gubernamentales con una visión amplia de acuerdo a los retos que el mundo globalizado exige.

\section{- MAESTRÍA EN SALUD PÚBLICA}

\section{DIRIGIDAA:}

Profesionales que se desempeñen en el sector Salud y otros campos de estudio, interesados en conocer el proceso de atención integral de salud en la población en general con un enfoque biopsicosocial, cultural y espiritual.

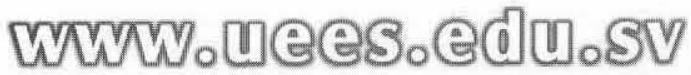 \\ PBX: ( 503$) 22754000$ \\ fAX: ( 503$) 22754040$}

